Pacific Journal of Mathematics

UNIFORM RANK OVER DIFFERENTIAL OPERATOR RINGS
AND POINCARÉ-BIRKHOFF-WITT EXTENIONS 


\title{
UNIFORM RANK OVER DIFFERENTIAL OPERATOR RINGS AND POINCARÉ-BIRKHOFF-WITT EXTENSIONS
}

\author{
A. D. Bell and K. R. Goodearl
}

This paper is principally concerned with the question of when a generalized differential operator ring $T$ over a ring $R$ must have the same uniform rank (Goldie dimension) or reduced rank as $R$, and with the corresponding questions for induced modules. In particular, when $R$ is either a right and left noetherian $\mathbb{Q}$-algebra, or a right noetherian right fully bounded $\mathbb{Q}$-algebra, it is proved that $T_{T}$ and $R_{R}$ have the same uniform rank. For any right noetherian ring $R$, it is proved that $T_{T}$ and $R_{R}$ have the same reduced rank. The type of generalized differential operator ring considered is any ring extension $T \supseteq R$ generated by a finite set of elements satisfying a suitable version of the PoincaréBirkhoff-Witt Theorem.

Although modules over differential operator rings have been extensively studied for some time, not much attention has been paid to questions of (uniform) rank, perhaps because the most natural relationship (that a differential operator ring should have the same rank as its coefficient ring) is not in general true. For polynomial rings, the relationship does hold, as proved by Shock [21, Theorem 2.6; 7, Theorem 3.23, p. 89]. Later partial results for a differential operator ring $T=R[\theta ; \delta]$ include the facts that if $M$ is a compressible right $R$-module then $M \otimes_{R} T$ is a compressible right $T$-module [10, Lemma 2.1], that if $M$ is a critical noetherian right $R$-module then $M \otimes_{R} T$ is a uniform right $T$-module [9, Lemma 4.6] and that if $R$ is a semiprime right noetherian ring then $T_{T}$ has the same rank as $R_{R}$ [22, Lemma 2.4].

The only previous general result along these lines of which we were initially aware is a recent result of Quinn [19, Theorem 15], who showed that if $R$ is a $\mathbb{Q}$-algebra and $\delta$ is locally nilpotent, then $T_{T}$ and $R_{R}$ have the same rank. That such a result cannot hold in positive characteristic is shown by the old example of $R=k[x] /\left(x^{2}\right)$ and $\delta=d / d x$, where $k$ is a field of characteristic 2 , in which case $R_{R}$ has rank 1 while $T_{T}$ has rank 2 [9, p. 851]. After our paper was first submitted, we saw a paper of Grzeszczuk, who proves that $T_{T}$ and $R_{R}$ have the same rank if $R$ is right nonsingular, or if $R$ is a $\mathbb{Q}$-algebra with the d.c.c. on right annihilators [12, Corollary 4]. 
In this paper, we show that various kinds of techniques yield sufficient conditions under which passing from $R$ to $T$ preserves the rank. For example, if $M$ is a nonsingular right $R$-module, or if each nonzero submodule of $M$ contains a nonzero element whose annihilator in $R$ is $\delta$-invariant, then $M \otimes_{R} T$ has the same rank as $M$. The latter result allows us to unify and slightly generalize the two steps in a result of Quinn [19, Theorem 14] and show that for any right module $M$ over any ring $R$, the induced module $M \otimes_{R} A_{1}(R)$ over the Weyl algebra $A_{1}(R)$ has the same rank as $M$. When $R$ is a right noetherian ring with no $\mathbb{Z}$-torsion which is tame as a right module over itself, we prove that $T_{T}$ has the same rank as $R_{R}$; in particular, this holds when $R$ is a right noetherian right fully bounded $\mathbb{Q}$-algebra, or when $R$ is a right noetherian $\mathbb{Q}$-algebra with the d.c.c. on right annihilators. To go along with the characteristic 2 example mentioned above, we construct a noetherian $\mathbb{Q}$-algebra $R$ with cyclic modules $M$ of rank 1 such that the induced modules $M \otimes_{R} T$ have arbitrarily large finite rank, and we construct a commutative $\mathbb{Q}$-algebra $R$ of rank 1 for which $T$ has rank $\infty$.

In order to study more general differential operator rings, including those smash/skew/twisted constructions in which a Lie algebra acts via derivations on a coefficient ring, without carrying around a large amount of notation and special conditions, we introduce the notion of a PoincaréBirkhoff-Witt extension of a ring $R$, meaning a ring extension $T \supseteq R$ generated by a (finite) set of elements for which a suitable PBW Theorem holds. Not all of the techniques used in the $R[\theta ; \delta]$ case seem applicable here, but, roughly speaking, those that are sufficiently noetherian can be adapted to PBW extensions. Thus we are able to prove that when $R$ is a right noetherian ring with no $\mathbb{Z}$-torsion which is tame as a right module over itself (and hence in the particular cases mentioned above), every PBW extension of $R$ has the same rank as $R$.

Finally, we consider the question of reduced rank, which is only defined in the noetherian case. Here we prove that any induced module $M \otimes_{R} T$ over a PBW extension $T$ of any right noetherian ring $R$ has the same reduced rank as $M$.

All rings and algebras in this paper are associative with unit, and all modules are unital right modules. The research of the second author was partially supported by a grant from the National Science Foundation.

0. Preliminaries. Let $M$ be a module over a ring $R$. The rank of $M$ (also known as the uniform rank, uniform dimension, Goldie rank, or Goldie dimension of $M$ ) is the supremum of those nonnegative integers $n$ such that $M$ contains an independent family of $n$ nonzero submodules. 
We denote the rank of $M$ by $\operatorname{rank}_{R}(M)$. Since by convention all our modules are right modules, $\operatorname{rank}_{R}(R)$ means the rank of $R_{R}$. Finite ranks may be computed using uniform submodules, for $M$ has finite rank if and only if $M$ contains independent uniform submodules $U_{1}, \ldots, U_{n}$ such that $U_{1} \oplus \cdots \oplus U_{n}$ is essential in $M$, in which case $\operatorname{rank}_{R}(M)=n[7$, Proposition 3.19 , p. 86].

Given a ring $R$ and a derivation $\delta$ on $R$, the formal linear differential operator ring $R[\theta ; \delta]$ is the overring of $R$ generated by $R$ and an element $\theta$ subject to the relations $\theta r=r \theta+\delta(r)$ for all $r \in R$. To abbreviate the condition that $R[\theta ; \delta]$ has been constructed from $R$ and $\delta$ in this fashion, we just write "let $R[\theta ; \delta]$ be a differential operator ring".

In a differential operator ring $T=R[\theta ; \delta]$, the powers of $\theta$ form a basis for $T$ as a free left (or right) $R$-module. Hence, any nonzero operator $t \in T$ may be uniquely expressed in the form

$$
t=t_{0}+t_{1} \theta+\cdots+t_{n} \theta^{n}
$$

for some $t_{i} \in R$ with $t_{n} \neq 0$. The integer $n$ is called the order of $t$, denoted $\operatorname{ord}(t)$, and the element $t_{n}$ is called the leading coefficient of $t$, denoted $\lambda(t)$. The zero element of $T$ is considered to have order $-\infty$ and leading coefficient 0 .

Note that $T$ may be filtered by order, and that then the associated graded ring $\operatorname{gr}(T)$ is a polynomial ring in one indeterminate over $R$. Thus if $R$ is right (left) noetherian, so is $T$.

As ${ }_{R} T$ is free, it is faithfully flat. Hence, given any right $R$-modules $N \leq M$, we may identify $N \otimes_{R} T$ with its image in $M \otimes_{R} T$. Observe that $M \otimes_{R} T$ is, as an abelian group, the direct sum of the subgroups $M \otimes \theta^{t}$ for $i=0,1, \ldots$. Thus any nonzero element $x \in M \otimes_{R} T$ may be uniquely expressed in the form

$$
x=\left(x_{0} \otimes 1\right)+\left(x_{1} \otimes \theta\right)+\cdots+\left(x_{n} \otimes \theta^{n}\right)
$$

for some $x_{i} \in M$ with $x_{n} \neq 0$. Following the established pattern, $n$ is called the order of $x$, denoted ord $(x)$, and $x_{n}$ is called the leading coefficient of $x$, denoted $\lambda(x)$. The zero element of $M \otimes_{R} T$ is considered to have order $-\infty$ and leading coefficient 0 .

The induced module $M \otimes_{R} T$ may be filtered by order, making it a filtered $T$-module, and its associated graded module is the $\operatorname{gr}(T)$-module induced from $M$. Thus if $M$ is a noetherian right $R$-module, $M \otimes_{R} T$ is a noetherian right $T$-module

If $\left\{M_{i}\right\}$ is any independent family of nonzero $R$-submodules of $M$, then $\left\{M_{i} \otimes_{R} T\right\}$ is an independent family of nonzero $T$-submodules of 
$M \otimes_{R} T$. Consequently,

$$
\operatorname{rank}_{T}\left(M \otimes_{R} T\right) \geq \operatorname{rank}_{R}(M),
$$

and in proving the reverse inequality $M$ may clearly be assumed to have finite rank.

The notation $N \leq_{e} M$ is used to mean that $N$ is an essential submodule of $M$. For any $x \in M$, the annihilator of $x$ in the ring $R$ is denoted $\operatorname{ann}_{R}(x)$. Given an ideal $I$ in $R$, we denote by $\mathscr{C}(I)$ the set of those $r \in R$ for which $r+I$ is a regular element (non-zero-divisor) in $R / I$.

1. Nonsingular modules. The main result of this section is that for an arbitrary differential operator ring $R[\theta ; \delta]$ and an arbitrary nonsingular right $R$-module $M$, the induced module $M \otimes_{R} R[\theta ; \delta]$ has the same rank as $M$. In the noetherian case, this will be used to deal with torsionfree modules over prime factor rings of $R$. When working with nonsingular modules, we may use the following sort of extensions in place of essential extensions.

Recall that an $R$-module $M$ is a rational extension of a submodule $N$, denoted $N \leq_{r} M$, provided that $\operatorname{Hom}_{R}(L / N, M)=0$ for any submodule $L$ of $M$ that contains $N$. Equivalently, if these are right modules, $N \leq_{r} M$ if and only if whenever $x, y \in M$ with $x \neq 0$, there exists $r \in R$ such that $x r \neq 0$ and $y r \in N$ [7, Proposition 2.25, p. 55].

Clearly any rational extension is also an essential extension, but the converse fails. For instance, $\mathbb{Z} / 4 \mathbb{Z}$ is an essential extension of $2 \mathbb{Z} / 4 \mathbb{Z}$ but not a rational extension. Our interest in rational extensions stems from the fact that any essential extension of nonsingular modules is a rational extension [7, Lemma 2.24, p. 55].

LEMMA 1.1. Let $T=R[\theta ; \delta]$ be a differential operator ring. If $N \leq{ }_{r} M$ are right $R$-modules, then $N \otimes_{R} T \leq{ }_{r} M \otimes_{R} T$ as $R$-modules and hence also as T-modules.

Proof. Consider $x, y \in M \otimes_{R} T$ with $x \neq 0$, and write

$$
\begin{aligned}
& x=\left(x_{0} \otimes 1\right)+\left(x_{1} \otimes \theta\right)+\cdots+\left(x_{m} \otimes \theta^{m}\right), \\
& y=\left(y_{0} \otimes 1\right)+\left(y_{1} \otimes \theta\right)+\cdots+\left(y_{n} \otimes \theta^{n}\right),
\end{aligned}
$$

where $x_{i}, y_{j} \in M$ and $x_{m} \neq 0$. For $k=n, n-1, \ldots, 0$, we show that there exists $r_{k} \in R$ such that $x_{m} r_{k} \neq 0$ and

$$
y r_{k} \in(M \otimes 1)+\cdots+\left(M \otimes \theta^{k-1}\right)+\left(N \otimes \theta^{k}\right)+\cdots+\left(N \otimes \theta^{n}\right) .
$$


First, since $N \leq{ }_{r} M$, there exists $r_{n} \in R$ such that $x_{m} r_{n} \neq 0$ and $y_{n} r_{n} \in N$, whence

$$
y r_{n} \in(M \otimes 1)+\cdots+\left(M \otimes \theta^{n-1}\right)+\left(N \otimes \theta^{n}\right) .
$$

Now let $0<k \leq n$ and assume that there exists $r_{k} \in R$ with the required properties. Write

$$
y r_{k}=\left(z_{0} \otimes 1\right)+\left(z_{1} \otimes \theta\right)+\cdots+\left(z_{n} \otimes \theta^{n}\right)
$$

with $z_{0}, \ldots, z_{k-1} \in M$ and $z_{k}, \ldots, z_{n} \in N$. There exists $s \in R$ such that $x_{m} r_{k} s \neq 0$ and $z_{k-1} s \in N$. Then the element $r_{k-1}=r_{k} s$ has the required properties, completing the induction step.

Thus $x_{m} r_{0} \neq 0$, whence $x r_{0} \neq 0$, and $y r_{0} \in N \otimes_{R} T$. Therefore $N \otimes_{R} T \leq{ }_{r} M \otimes_{R} T$ as $R$-modules. It follows immediately that $N \otimes_{R} T \leq{ }_{r} M \otimes_{R} T$ as $T$-modules.

LEMMA 1.2. Let $N \leq_{r} M$ be right $R$-modules. Given $x_{1}, x_{2}, y \in M$ with each $x_{i} \neq 0$, there exists $r \in R$ such that each $x_{i} r \neq 0$ and $y r \in N$.

Proof. There exist $r_{1}, r_{2} \in R$ such that $x_{i} r_{i} \neq 0$ and $y r_{i} \in N$. If $x_{2} r_{1} \neq 0$ set $r=r_{1}$, while if $x_{1} r_{2} \neq 0$ set $r=r_{2}$. If $x_{2} r_{1}=x_{1} r_{2}=0$, set $r=r_{1}+r_{2}$.

Proposition 1.3. Let $T=R[\theta ; \delta]$ be a differential operator ring, and let $M$ be a right $R$-module. If $M$ is a rational extension of each of its nonzero submodules, then $M \otimes_{R} T$ is a rational T-module extension of each of its nonzero T-submodules.

Proof. It suffices to show that $z T \leq{ }_{r} M \otimes_{R} T$ for each nonzero $z \in M \otimes_{R} T$. Hence, given $x, y \in M \otimes_{R} T$ with $x \neq 0$, we need to find $s, t \in T$ such that $x s \neq 0$ and $y s=z t$. Of course if $y=0$ we may use $s=1$ and $t=0$. Thus it suffices to prove that for any nonzero $x, y, z \in$ $M \otimes_{R} T$, there exist $s, t \in T$ such that $x s \neq 0$ and $y s=z t$. We actually prove the following stronger property:

(*) Given any nonzero $x, y, z \in M \otimes_{R} T$, there exist $s, t \in T$ such that $\lambda(x) \lambda(s) \neq 0$ and $y s=z t$, while also $\operatorname{ord}(s)-\operatorname{ord}(t) \geq$ $\operatorname{ord}(z)-\operatorname{ord}(y)$. (Here $s$ must be nonzero, whence $\operatorname{ord}(s) \geq 0$. We allow the possibility that $t=0$, in which case ord $(s)-\operatorname{ord}(t)=\infty$.)

Set $m=\operatorname{ord}(y)$ and $n=\operatorname{ord}(z)$; we induct on $m+n$.

If $m+n=0$, then $y=y_{0} \otimes 1$ and $z=z_{0} \otimes 1$ for some nonzero $y_{0}, z_{0} \in M$. Since $\lambda(x) \neq 0$ and $z_{0} R \leq{ }_{r} M$, there exist $s, t \in R$ such that $\lambda(x) s \neq 0$ and $y_{0} s=z_{0} t$. Then $y s=z t$ and $\operatorname{ord}(s)-\operatorname{ord}(t) \geq 0=$ $n-m$. 
Now let $m+n>0$, and assume that $(*)$ holds in all cases where $\operatorname{ord}(y)+\operatorname{ord}(z)<m+n$. Since $\lambda(x), \lambda(y), \lambda(z)$ are nonzero elements of $M$ and $\lambda(z) R \leq{ }_{r} M$, Lemma 1.2 provides us with elements $p, q \in R$ such that $\lambda(x) p \neq 0$ and $\lambda(y) p \neq 0$ while $\lambda(y) p=\lambda(z) q$. Hence, $x p$, $y p, z q$ are all nonzero, ord $(y p)=m$ and $\operatorname{ord}(z q)=n$, and $\lambda(y p)=\lambda(z q)$. If there exist $s^{\prime}, t^{\prime} \in T$ such that $\lambda(x p) \lambda\left(s^{\prime}\right) \neq 0$ and $y p s^{\prime}=z q t^{\prime}$ while also ord $\left(s^{\prime}\right)-\operatorname{ord}\left(t^{\prime}\right) \geq n-m$, then $\lambda(x) p \lambda\left(s^{\prime}\right) \neq 0$ and so $\lambda\left(p s^{\prime}\right)=$ $p \lambda\left(s^{\prime}\right)$. Consequently, $\lambda(x) \lambda\left(p s^{\prime}\right) \neq 0$ and

$$
\operatorname{ord}\left(p s^{\prime}\right)-\operatorname{ord}\left(q t^{\prime}\right) \geq \operatorname{ord}\left(s^{\prime}\right)-\operatorname{ord}\left(t^{\prime}\right) \geq n-m,
$$

proving $(*)$.

Thus we may assume, without loss of generality, that $\lambda(y)=\lambda(z)$.

First, suppose that $m \geq n$. If $y=z \theta^{m-n}$, we may use $s=1$ and $t=\theta^{m-n}$. Otherwise, $y-z \theta^{m-n}$ is nonzero and has order less than $m$. By the induction hypothesis, there exist $s, t^{\prime} \in T$ such that $\lambda(x) \lambda(s) \neq 0$ and $\left(y-z \theta^{m-n}\right) s=z t^{\prime}$, while also

$$
\operatorname{ord}(s)-\operatorname{ord}\left(t^{\prime}\right) \geq \operatorname{ord}(z)-\operatorname{ord}\left(y-z \theta^{m-n}\right)>n-m .
$$

Then $y s=z\left(\theta^{m-n} s+t^{\prime}\right)$. Since $\operatorname{ord}\left(\theta^{m-n} s\right)=m-n+\operatorname{ord}(s)>\operatorname{ord}\left(t^{\prime}\right)$, we see that

$$
\operatorname{ord}\left(\theta^{m-n} s+t^{\prime}\right)=m-n+\operatorname{ord}(s),
$$

whence ord $(s) \cdot \operatorname{ord}\left(\theta^{m-n} s+t^{\prime}\right)=n-m$.

Finally, suppose that $m<n$. If $z=y \theta^{n-m}$, we may use $s=\theta^{n-m}$ and $t=1$. Otherwise, $z-y \theta^{n-m}$ is nonzero and has order less than $n$. By the induction hypothesis, applied to the elements $x, z-y \theta^{n-m}, y$, there exist $t, s^{\prime} \in T$ such that $\lambda(x) \lambda(t) \neq 0$ and $\left(z-y \theta^{n-m}\right) t=y s^{\prime}$, while

$$
\operatorname{ord}(t)-\operatorname{ord}\left(s^{\prime}\right) \geq \operatorname{ord}(y)-\operatorname{ord}\left(z-y \theta^{n-m}\right)>m-n \text {. }
$$

Then $y\left(\theta^{n-m} t+s^{\prime}\right)=z t$. Since ord $\left(\theta^{n-m} t\right)=n-m+\operatorname{ord}(t)>\operatorname{ord}\left(s^{\prime}\right)$, we see that

$$
\operatorname{ord}\left(\theta^{n-m} t+s^{\prime}\right)=n-m+\operatorname{ord}(t),
$$

whence $\operatorname{ord}\left(\theta^{n-m} t+s^{\prime}\right)-\operatorname{ord}(t)=n-m$. In addition,

$$
\lambda(x) \lambda\left(\theta^{n-m} t+s^{\prime}\right)=\lambda(x) \lambda(t) \neq 0 .
$$

This verifies the induction step, and therefore $(*)$ is proved.

Observe that a nonzero module $M$ is monoform (i.e., every nonzero homomorphism between submodules of $M$ is a monomorphism) if and only if $M$ is a rational extension of each of its nonzero submodules. Hence, to rephrase Proposition 1.3, if $M$ is a monoform right $R$-module then $M \otimes_{R} T$ is a monoform right $T$-module. 
As an immediate application of Proposition 1.3, we can remove the noetherian hypothesis from [9, Lemma 4.6].

Corollary 1.4. Let $T=R[\theta ; \delta]$ be a differential operator ring. If $M$ is a critical (in the sense of Krull dimension) right $R$-module, then $M \otimes_{R} T$ is a uniform right T-module.

Proof. Since $M$ is nonzero, so is $M \otimes_{R} T$. If $N \leq L$ are nonzero submodules of $M$, then $L / N$ has smaller Krull dimension than any nonzero submodule of $M$, whence $\operatorname{Hom}_{R}(L / N, M)=0$. Thus $M$ is a rational extension of each of its nonzero submodules, and Proposition 1.3 applies. In particular, all nonzero $T$-submodules of $M \otimes_{R} T$ are essential in $M \otimes_{R} T$, whence $M \otimes_{R} T$ is uniform.

Proposition 1.5. Let $T=R[\theta ; \delta]$ be a differential operator ring. If $M$ is any nonsingular right $R$-module, then $\operatorname{rank}_{T}\left(M \otimes_{R} T\right)=\operatorname{rank}_{R}(M)$.

Proof. We may assume that $\operatorname{rank}_{R}(M)=n<\infty$. Then $M$ contains independent uniform submodules $U_{1}, \ldots, U_{n}$ such that $U_{1} \oplus \cdots \oplus U_{n} \leq_{e}$ $M$. Since $M$ is nonsingular, $U_{1} \oplus \cdots \oplus U_{n} \leq{ }_{r} M$, and each $U_{i}$ is a rational extension of all its nonzero submodules. By Lemma 1.1 and Proposition 1.3,

$$
\left(U_{1} \otimes_{R} T\right) \oplus \cdots \oplus\left(U_{n} \otimes_{R} T\right) \leq_{e} M \otimes_{R} T
$$

and each $U_{i} \otimes_{R} T$ is a uniform right $T$-module. Therefore $\operatorname{rank}_{T}\left(M \otimes_{R} T\right)$ $=n$.

Corollary 1.6. Let $T=R[\theta ; \delta]$ be a differential operator ring. If $R$ is a semiprime right Goldie ring and $M$ is a torsionfree right $R$-module, then $\operatorname{rank}_{T}\left(M \otimes_{R} T\right)=\operatorname{rank}_{R}(M)$.

Using different methods, the case $M=R$ of Proposition 1.5 has also been proved by Grzeszczuk [12, Corollary 4], and the case $M=R$ with $R$ right noetherian of Corollary 1.6 was proved by Sigurdsson [22, Lemma 2.4].

The case of Proposition 1.5 in which $R$ is right noetherian may be proved without the use of Proposition 1.3, as we will see in a more general setting in the proof of Proposition 5.4.

2. Tame modules and the noetherian case. In this section we prove that the uniform rank of a ring and its differential operator ring are the same if the ring is (right and left) noetherian and has no $\mathbb{Z}$-torsion. We 
prove this by first proving some more general results about induced modules.

Let $R$ be a right noetherian ring and let $U$ be a uniform right $R$-module. Then there is a unique prime ideal $P$ of $R$ which is the largest annihilator of any nonzero submodule of $U$. This prime ideal is called the assassinator of $U$, and we say that $U$ is tame if it contains a copy of a nonzero right ideal of $R / P$. Alternatively, $U$ is tame if and only if the submodule $\operatorname{ann}_{U}(P)$ is torsionfree as an $(R / P)$-module. An arbitrary right $R$-module $M$ is tame if all of its uniform submodules are tame, and we denote the set of assassinator prime ideals of uniform submodules of $M$ by ass $(M)$. It is not hard to see that neither the tameness of $M$ nor the set ass $(M)$ is changed by passing to an essential extension or an essential submodule of $M$.

Proposition 2.1. Let $T=R[\theta ; \delta]$ be a differential operator ring, where $R$ is a right noetherian ring, and let $M$ be a tame right $R$-module such that each member of ass $(M)$ is $\delta$-invariant. Then

$$
\operatorname{rank}_{R}(M)=\operatorname{rank}_{T}\left(M \otimes_{R} T\right) .
$$

Proof. Let $E$ be the injective hull of $M$. Then we know that

$$
\operatorname{rank}_{R}(E)=\operatorname{rank}_{R}(M) \leq \operatorname{rank}_{T}\left(M \otimes_{R} T\right) \leq \operatorname{rank}_{T}\left(E \otimes_{R} T\right),
$$

and so we need only show that $\operatorname{rank}_{T}\left(E \otimes_{R} T\right)=\operatorname{rank}_{R}(E)$. As noted above, $E$ is tame and all of its assassinator prime ideals are $\delta$-invariant. As $R$ is right noetherian, $E$ is a direct sum of uniform injective modules; since the tensor product respects direct sums, it is clearly enough to do the case where $E$ is uniform.

Our hypotheses now imply that there is a $\delta$-invariant prime ideal $P$ of $R$ such that $E_{0}=\operatorname{ann}_{E}(P)$ is a torsionfree uniform right $(R / P)$-module. Note that $P T$ is an ideal of $T$ and that $T / P T \cong(R / P)\left[\theta ; \delta^{\prime}\right]$ where $\delta^{\prime}$ is the derivation induced on $R / P$ by $\delta$. Thus Corollary 1.6 implies that the $T$-module

$$
E_{0} \otimes_{R} T \cong E_{0} \otimes_{R / P}(T / P T)
$$

is uniform. To finish the proof, we must show $E_{0} \otimes_{R} T$ is an essential submodule of $E \otimes_{R} T$.

If this is not true, there is a nonzero $a \in E \otimes_{R} T$ such that $a T \cap\left(E_{0} \otimes_{R} T\right)=0$. Pick such an $a$ of the form

$$
a=\left(a_{0} \otimes 1\right)+\left(a_{1} \otimes \theta\right)+\cdots+\left(a_{n} \otimes \theta^{n}\right)
$$


with minimal order $n$, and so $a_{n} \neq 0$. Since $E_{0}$ is essential in $E$, there exists an $r \in R$ such that $a_{n} r \in E_{0}$ and $a_{n} r \neq 0$. We may replace $a$ by $a r$, and hence without loss of generality we may suppose that $a_{n}$ is in $E_{0}$. Thus $a_{n} P=0$, and since $P$ is $\delta$-invariant, it is easy to see that $\left(a_{n} \otimes \theta^{n}\right) P$ $=0$. Since $a P T \cap\left(E_{0} \otimes_{R} T\right)=0$, the minimality of $n$ implies $a P=0$. Thus $\left(a-\left(a_{n} \otimes \theta^{n}\right)\right) P=0$ and so $a_{n-1} P=0$. Using the $\delta$-invariance of $P$ and repeating the above arguments, it is now easy to see that each $a_{l} P=0$. But this implies that $a \in E_{0} \otimes_{R} T$, which contradicts our assumption that $a \neq 0$.

In order to use the last result to determine the ranks of differential operator rings, we make the following observation. We thank the referee for a simplification of our original proof.

LEMMA 2.2. If $R$ is a ring with no $\mathbb{Z}$-torsion and with the a.c.c. on annihilator ideals, and $\delta$ is a derivation on $R$, then any prime annihilator ideal in $R$ is $\delta$-invariant.

Proof. Let $P$ be a prime ideal in $R$ such that $P=r$-ann $(X)$ for some subset $X$ of $R$. (The proof is essentially the same if $P=l$-ann $(X)$.) Since $R$ has the a.c.c. on annihilator ideals, there is a positive integer $n$ such that

$$
l \text {-ann }\left(P^{n}\right)=l \text {-ann }\left(P^{n+1}\right)=\cdots .
$$

Set $I=l-\operatorname{ann}\left(P^{n}\right)$, and observe that

$$
\delta(I) P^{n+1} \subseteq \delta\left(I P^{n+1}\right)-I \delta\left(P^{n+1}\right) \subseteq \delta\left(I P^{n+1}\right)-I P^{n}=0,
$$

whence $\delta(I) \subseteq I$, and consequently $r$-ann $(I)$ is $\delta$-invariant. Since $X \subseteq I$, we have

$$
P^{n} \subseteq r-\operatorname{ann}(I) \subseteq r-\operatorname{ann}(X)=P,
$$

and thus $P$ is the unique prime ideal minimal over $r$-ann $(I)$. As $R$ has no $\mathbb{Z}$-torsion, it is clear that $R / r$-ann $(I)$ has no $\mathbb{Z}$-torsion. Therefore [11, Proposition 1.3] implies that $P$ is $\delta$-invariant.

The main result of this section is an immediate corollary of the two preceding results.

THEOREM 2.3. Let $T=R[\theta ; \delta]$ be a differential operator ring, where $R$ is a right noetherian ring with no $\mathbb{Z}$-torsion. If $R$ is tame as a right module over itself, then $\operatorname{rank}_{R}(R)=\operatorname{rank}_{T}(T)$. 
COROLlaRy 2.4. Let $T=R[\theta ; \delta]$ be a differential operator ring, where $R$ is a right noetherian ring with no $\mathbb{Z}$-torsion. If either $R$ is right fully bounded or $R$ has the d.c.c. on right annihilators, then $\operatorname{rank}_{R}(R)=$ $\operatorname{rank}_{T}(T)$.

Proof. In each case, we observe that the ring $R$ is tame as a right module over itself and hence the theorem applies. Tameness is immediate in case $R$ is right fully bounded, since a finitely generated torsion module over a bounded prime ring is always unfaithful, and hence all right $R$-modules are tame in this case.

Alternatively, one can handle both cases at once by first noting that if $I$ is a right ideal of $R$, there exist $x_{1}, \ldots, x_{n} \in I$ such that

$$
r \text {-ann }(I)=r \text {-ann }\left(x_{1}\right) \cap \cdots \cap r \text {-ann }\left(x_{n}\right) .
$$

This is well-known to hold (for any right $R$-module $I$ ) in the fully bounded case. In the other case, we take $\left\{x_{1}, \ldots, x_{n}\right\}$ to be a finite subset of $I$ whose right annihilator is as small as possible.

Now suppose $I$ is a uniform right ideal of $R$ such that $P=r$-ann $(I)$ is prime, and let $x_{1}, \ldots, x_{n}$ be as in the last paragraph. If $I$ is not torsionfree as an $(R / P)$-module, then it is torsion and so each $x_{i}$ is annihilated by some element of $\mathscr{C}(P)$. Using the right common multiple property of the elements of $\mathscr{C}(P)$ in $R / P$, we find that there is an element $c \in \mathscr{C}(P)$ such that $x_{i} c=0$ for each $i$. Thus $I c=0$, which contradicts $r$-ann $(I)=P$. This shows $I$ must be torsionfree as a right $(R / P)$-module, and so $I$ is tame.

Since the d.c.c. on right annihilators is equivalent to the a.c.c. on left annihilators, and either condition is inherited by subrings, it follows that $\operatorname{rank}_{R}(R)=\operatorname{rank}_{T}(T)$ if $R$ is a right noetherian ring with no $\mathbb{Z}$-torsion which embeds in either a right artinian or left noetherian ring. In particular, the rank equality holds for a right and left noetherian ring with no $\mathbb{Z}$-torsion.

The second case of Corollary 2.4 holds even if $R$ is not right noetherian [12, Corollary 4].

3. Non-noetherian methods. In [19, Theorem 14], Quinn showed that the Weyl algebra $A_{1}(R)$ over any ring $R$ has the same rank as $R$, using Shock's proof of the polynomial ring case [21, Theorem 2.6; 7, Theorem 3.23, p. 89] to get from $R$ to $R[x]$ and a modified version of Shock's argument to get from $R[x]$ to $R[x][\theta ; d / d x]$. Here we develop a generalization which covers both of these steps simultaneously. 
LEMma 3.1. Let $T=R[\theta ; \delta]$ be a differential operator ring, let $M$ be a right $R$-module, and let $A$ be a nonzero T-submodule of $M \otimes_{R} T$. If $x$ is a nonzero element of $A$ of minimal order, then $\operatorname{ann}_{T}(x)=\operatorname{ann}_{R}(\lambda(x)) T$. Thus $x T \cong \lambda(x) R \otimes_{R} T$ as right $T$-modules.

Proof. This is a standard minimal order argument. For details, see for example the proof of [9, Lemma 4.1].

Lemma 3.2. Let $T=R[\theta ; \delta]$ be a differential operator ring, and let $N$ be a right $R$-module. Assume that each nonzero submodule of $N$ contains a nonzero element whose annihilator in $R$ is invariant under $\delta+$ a for some $a \in R$.

(a) If $M$ is any essential extension of $N$, then $M \otimes_{R} T$ is an essential $T$-module extension of $N \otimes_{R} T$.

(b) If $N$ is uniform, then $N \otimes_{R} T$ is a uniform right $T$-module.

Proof. (a) If not, there is a nonzero element $x \in M \otimes_{R} T$ such that $x T \cap\left(N \otimes_{R} T\right)=0$, and we may assume that $n=\operatorname{ord}(x)$ is minimal for this. Since $N \leq_{e} M$, there exists $r \in R$ such that $\lambda(x) r$ is a nonzero element of $N$, and we may replace $x$ by $x r$. Thus there is no loss of generality in assuming that $\lambda(x) \in N$.

By hypothesis, there exist $s, a \in R$ such that $\lambda(x) s \neq 0$ and $\operatorname{ann}_{R}(\lambda(x) s)$ is invariant under $\delta+a$. Since we may replace $x$ by $x s$, there is no loss of generality in assuming that

$$
(\delta+a)\left(\operatorname{ann}_{R}(\lambda(x))\right) \subseteq \operatorname{ann}_{R}(\lambda(x)),
$$

whence $(\theta+a)\left(\operatorname{ann}_{R}(\lambda(x))\right) \subseteq \operatorname{ann}_{R}(\lambda(x)) T$. As $x t T \cap\left(N \otimes_{R} T\right)=0$ for all $t \in T$, the minimality of $n$ implies that all nonzero elements of $x T$ have order at least $n$. Hence, $\operatorname{ann}_{T}(x)=\operatorname{ann}_{R}(\lambda(x)) T$, by Lemma 3.1. Thus

$$
(\theta+a)\left(\operatorname{ann}_{T}(x)\right) \subseteq \operatorname{ann}_{T}(x)=\operatorname{ann}_{R}(\lambda(x)) T .
$$

Set $z=x-\left(\lambda(x) \otimes(\theta+a)^{n}\right)$, and note that $z \neq 0$, because $x \notin$ $N \otimes_{R} T$. Since $z$ has order less than $n$, we have $z T \cap\left(N \otimes_{R} T\right) \neq 0$ by the minimality of $n$, and so there exists $p \in T$ such that $z p$ is a nonzero element of $N \otimes_{R} T$. Then $x p \in N \otimes_{R} T$, whence $x p=0$. Now $p \in$ $\operatorname{ann}_{T}(x)$, and consequently

$$
(\theta+a)^{n} p \in \operatorname{ann}_{R}(\lambda(x)) T .
$$

However, from $\lambda(x) \otimes(\theta+a)^{n} p=0$ we obtain $z p=0$, a contradiction. 
Therefore $N \otimes_{R} T \leq{ }_{e} M \otimes_{R} T$.

(b) If not, there exist nonzero $x, y \in N \otimes_{R} T$ such that $x T \cap y T=0$. Set $n=\operatorname{ord}(x)$ and $m=\operatorname{ord}(y)$. We may assume that $n+m$ is minimal, and that $n \geq m$. As $N$ is uniform, there exist $r, s \in R$ such that $\lambda(x) r=$ $\lambda(y) s \neq 0$, and we may replace $x, y$ by $x r, y s$. Thus we may assume, without loss of generality, that $\lambda(x)=\lambda(y)$.

As in the proof of (a), we may also assume that there exists $a \in R$ for which

$$
(\theta+a)\left(\operatorname{ann}_{T}(x)\right) \subseteq \operatorname{ann}_{T}(x)=\operatorname{ann}_{R}(\lambda(x)) T=\operatorname{ann}_{T}(y) .
$$

Now let $z=x-y(\theta+a)^{n-m}$, and note that $z \neq 0$, because $x \notin y T$. Since $z$ has order less than $n$, we have $z T \cap y T \neq 0$ by the minimality of $n+m$, and so there exist $p, q \in T$ such that $z p=y q \neq 0$. As a result, $x p \in y T$, whence $x p=0$. However, we then obtain

$$
(\theta+a)^{n-m} p \in \operatorname{ann}_{T}(y)
$$

and hence $z p=0$, a contradiction.

Therefore $N \otimes_{R} T$ is uniform.

Proposition 3.3. Let $T=R[\theta ; \delta]$ be a differential operator ring, and let $M$ be a right $R$-module. Assume that each nonzero submodule of $M$ contains a nonzero element whose annihilator in $R$ is invariant under $\delta+a$ for some $a \in R$. Then $\operatorname{rank}_{T}\left(M \otimes_{R} T\right)=\operatorname{rank}_{R}(M)$.

Proof. We may assume that $\operatorname{rank}_{R}(M)=n<\infty$. Then $M$ contains independent uniform submodules $U_{1}, \ldots, U_{n}$ such that $U_{1} \oplus \cdots \oplus U_{n} \leq_{e}$ $M$. By Lemma 3.2, each $U_{l} \otimes_{R} T$ is uniform, and $\oplus\left(U_{i} \otimes_{R} T\right) \leq_{e}$ $M \otimes_{R} T$. Therefore $\operatorname{rank}_{T}\left(M \otimes_{R} T\right)=n$.

As an application of Proposition 3.3, we obtain the following generalization of Quinn's result [19, Theorem 14].

THEOREM 3.4. Let $T=A_{1}(R)$ for some ring $R$, and let $M$ be any right $R$-module. Then $\operatorname{rank}_{T}\left(M \otimes_{R} T\right)=\operatorname{rank}_{R}(M)$.

Proof. We may write $T=S[\theta ; d / d x]$ where $S=R[x]$ is a polynomial ring over $R$. Since $S$ may be viewed as a differential operator ring formed using the zero derivation, the hypotheses of Proposition 3.3 are trivially satisfied, and hence $\operatorname{rank}_{S}\left(M \otimes_{R} S\right)=\operatorname{rank}_{R}(M)$. To use Proposition 3.3 to obtain $\operatorname{rank}_{T}\left(M \otimes_{R} T\right)=\operatorname{rank}_{S}\left(M \otimes_{R} S\right)$, it suffices to show that any 
nonzero $S$-submodule $A$ of $M \otimes_{R} S$ contains a nonzero element whose annihilator in $S$ is invariant under $d / d x$.

Just choose a nonzero element $y \in A$ of minimal degree (order), remembering that $M \otimes_{R} S=M \otimes_{R} R[x]$. By Lemma 3.1,

$$
\operatorname{ann}_{S}(y)=\operatorname{ann}_{R}(\lambda(y)) S=\operatorname{ann}_{R}(\lambda(y))[x],
$$

which is clearly invariant under $d / d x$.

Proposition 3.3 may also be used in case $R$ is a commutative noetherian ring with no $\mathbb{Z}$-torsion, in place of Corollary 2.4. For if $I$ is any nonzero ideal of $R$ and $x \in I$ is chosen with $\operatorname{ann}_{R}(x)$ prime, then $\operatorname{ann}_{R}(x)$ is $\delta$-invariant by Lemma 2.2 . Hence, the equality of $\operatorname{rank}_{T}(T)$ and $\operatorname{rank}_{R}(R)$ in this case follows from the proposition.

A final, easy case in which the hypotheses of Proposition 3.3 hold is that in which $\delta^{2}=0$ and $1 / 2 \in R$. It follows easily that $\delta(r) \delta(s)=0$ for all $r, s \in R$. Given a nonzero submodule $N \leq M$, choose a nonzero element $x \in N$. If $\operatorname{ann}_{R}(x)$ is $\delta$-invariant, fine. If not, there exists $r \in R$ such that $x r=0$ but $x \delta(r) \neq 0$. Then $x \delta(r) \delta(s)=0$ for all $s \in R$, whence $\operatorname{ann}_{R}(x \delta(r))$ is $\delta$-invariant.

4. Examples. The easiest examples of differential operator rings which have greater rank than their coefficient rings occur in characteristic $p>0$. For instance, let $k$ be a field of characteristic $p$, let $R=k[x] /\left(x^{p}\right)$, and let $y$ denote the coset of $x$ in $R$. Then $\operatorname{rank}_{R}(R)=1$. Since $(d / d x)\left(x^{p}\right)=0$ in characteristic $p$, the derivation $d / d x$ induces a $k$-linear derivation $\delta$ on $R$ such that $\delta(y)=1$. In the differential operator ring $T=R[\theta ; \delta]$, one easily checks that the operator $y^{p-1} \theta^{p-1} /(p-1)$ ! is a nontrivial idempotent, whence $\operatorname{rank}_{T}(T) \geq 2$. [Actually, $\operatorname{rank}_{T}(T)=p$ : this will follow from Theorem 6.4, which says that $T_{T}$ has the same reduced rank as $R_{R}$, namely $p$. For $R$ is $\delta$-simple, whence $T$ is prime, and thus the reduced rank of $T_{T}$ is just $\operatorname{rank}_{T}(T)$.]

In characteristic zero, we first construct examples of modules whose ranks enlarge when induced up to differential operator rings.

EXAMPLE 4.1. Let $R=k[x]$ and $T=A_{1}(k)=k[x][\theta ; d / d x]$ where $k$ is a field of characteristic zero. For each positive integer $n$, the $k[x]$-module $M_{n}=k[x] /\left(x^{n}\right)$ has rank 1 , whereas the $T$-module $M_{n} \otimes_{R} T \cong T / x^{n} T$ has rank $n$.

Proof. Obviously $\operatorname{rank}_{R}\left(M_{n}\right)=1$ for all $n$. Since $M_{1}$ is a simple $k[x]$-module, Corollary 1.4 shows that $M_{1} \otimes_{R} T \cong T / x T$ is a uniform $T$-module. (Actually, it is easy to check that $T / x T$ is a simple $T$-module.) 
We shall prove that $T / x^{n} T \cong(T / x T)^{n}$ for all $n$, from which it is

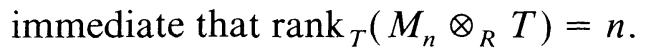

For $n=1$, there is nothing to prove. For the induction step, we show that

$$
T / x^{n+1} T \cong(T / x T) \oplus\left(T / x^{n} T\right),
$$

for which it suffices to see that the short exact sequence

$$
0 \rightarrow T / x T \stackrel{\left(x^{n}\right)^{*}}{\longrightarrow} T / x^{n+1} T \stackrel{\pi}{\rightarrow} T / x^{n} T \rightarrow 0
$$

splits, where $\left(x^{n}\right)^{*}$ is the map induced by left multiplication by $x^{n}$, and $\pi$ is the quotient map. By [8, Lemma 2.9], this sequence splits if and only if there exist $u, v \in T$ such that $u x^{n}+x v=1$. However, such elements are easy to find: observing that $\theta^{n} x^{n}=n !+x w$ for some $w \in T$, we conclude that

$$
\left(\theta^{n} / n !\right) x^{n}+x(-w / n !)=1
$$

To build a ring example, we incorporate the polynomial ring $k[x]$ and all the modules $k[x] /\left(x^{n}\right)$ within one ring, as follows.

EXAMPLE 4.2. There exists a commutative $\mathbb{Q}$-algebra $R$ with a derivation $\delta$ such that $R_{R}$ has rank 1 whereas the differential operator ring $T=R[\theta ; \delta]$ has infinite rank over itself.

Proof. Set $S=k[x]$ where $k$ is a field of characteristic zero, and let $M$ denote the $S$-module $k\left[x, x^{-1}\right] / S$. Then let $R$ be the trivial extension of $S$ by $M$, that is, $R=S \times M$ as an abelian group, with multiplication given by the rule $(s, m)\left(s^{\prime}, m^{\prime}\right)=\left(s s^{\prime}, s m^{\prime}+s^{\prime} m\right)$. Observe that $R$ is a commutative $\mathbb{Q}$-algebra, and that $I=0 \times M$ is an ideal of $R$. Since $M$ is a faithful uniform $S$-module, $I$ is an essential uniform ideal of $R$, whence $\operatorname{rank}_{R}(R)=1$.

Let $d$ denote the derivation $d / d x$ on both $S$ and $k\left[x, x^{-1}\right]$. Then $d$ induces an additive endomorphism $d_{M}$ on $M$, and we observe that $d_{M}(s m)=d(s) m+s d_{M}(m)$ for all $s \in S$ and $m \in M$. Set $\delta(s, m)=$ $\left(d(s), d_{M}(m)\right)$ for all $(s, m) \in R$, and observe that $\delta$ is a derivation on $R$.

Now $I$ is a $\delta$-ideal of $R$, whence $I T$ is an ideal of $T$ and

$$
T / I T \cong(R / I)[\theta ; \delta] \cong A_{1}(k) .
$$

Since $I^{2}=0$, we similarly have

$$
I T \cong I \otimes_{R} T \cong I \otimes_{R / I}(T / I T) \cong M \otimes_{S} A_{1}(k) .
$$


As $M$ contains copies of the $S$-modules $S / x^{n} S$, for all $n \in \mathbb{N}$, Example 4.1 shows that $M \otimes_{S} A_{1}(k)$ has rank $\infty$ as an $A_{1}(k)$-module. Therefore $\operatorname{rank}_{T}(I T)=\infty$, whence $\operatorname{rank}_{T}(T)=\infty$.

We know of no example of a right noetherian $\mathbb{Q}$-algebra $R$ with a derivation $\delta$ for which $R[\theta ; \delta]$ has greater rank than $R$.

5. Poincaré-Birkhoff-Witt extensions. A number of generalized differential operator rings in the literature, with names like smashed/skew enveloping rings, are formed from a Lie ring $L$ acting as derivations on a ring $R$. A common feature of these constructions is that under suitable hypotheses, such as a projective module structure on $L$ compatible with the action of $L$ on $R$, a version of the Poincaré-Birkhoff-Witt Theorem holds. Since much of the work on such rings follows from the form given by the PBW Theorem, we find it much more convenient to take the PBW form as our starting point, thus avoiding the necessity of carrying along the notation of $L, R$, the action of $L$ on $R$, etc. in all later results.

We say that an overring $T$ of a ring $R$ is a (finite) Poincaré-BirkhoffWitt extension of $R$ (hereafter called a $P B W$ extension, for short) if there exist elements $\theta_{1}, \ldots, \theta_{n} \in T$ such that

(a) The ordered monomials $\theta_{1}^{i(1)} \theta_{2}^{\prime(2)} \cdots \theta_{n}^{i(n)}$ (for nonnegative integers $i(1), \ldots, i(n))$ form a basis for $T$ as a free left $R$-module;

(b) $\left[\theta_{i}, R\right] \subseteq R$ for each $i=1, \ldots, n$;

(c) $\left[\theta_{i}, \theta_{j}\right] \in R+R \theta_{1}+\cdots+R \theta_{n}$ for all $i, j=1, \ldots, n$.

(An infinite PBW extension would be formed in a similar manner, using a well-ordered set of elements $\theta_{i}$.) It follows from (a), (b) that the ordered monomials $\theta_{1}^{i(1)} \theta_{2}^{i(2)} \ldots \theta_{n}^{i(n)}$ also form a basis for $T$ as a free right $R$-module, and it follows from (b) that

$$
R+R \theta_{1}+\cdots+R \theta_{n}=R+\theta_{1} R+\cdots+\theta_{n} R .
$$

Thus our definition is left-right symmetric.

To abbreviate conditions (a), (b), (c), we shall just write "let $T=$ $R\left[\theta_{1}, \ldots, \theta_{n}\right]$ be a PBW extension".

As examples of PBW extensions, we mention: (a) the enveloping algebra of any finite-dimensional Lie algebra; (b) any differential operator ring $R\left[\theta_{1}, \ldots, \theta_{n} ; \delta_{1}, \ldots, \delta_{n}\right]$ formed from commuting derivations $\delta_{1}, \ldots, \delta_{n}$ on $R$; (c) any Weyl algebra $A_{n}(R)$ (viewed as an extension of $R$ by $2 n$ elements); (d) those differential operator rings $V(R, L)$ introduced by Rinehart [20, p. 197] (see also [13, p. 396], [4], [5]) where $L$ is a Lie algebra which is also a (finitely generated) free $R$-module equipped with a suitable Lie algebra map to derivations on $R$ (a more general PBW Theorem is 
obtained here after assuming that $L$ is a projective $R$-module [20, Theorem 3.1]); (e) the twisted or smash product differential operator ring $R \#_{\sigma} U(\mathrm{~g})$ studied by McConnell [16, Theorem 2.8; 17, p. 190], Chin [2], and others, where $\mathfrak{g}$ is a finite-dimensional Lie algebra acting on $R$ by derivations, and $\sigma$ is a Lie 2-cocycle with values in $R$; (f) the universal enveloping rings $U(V, R, K)$ recently introduced by Passman [18], where $K$ is a field, $R$ is a $K$-algebra, and $V$ is a $K$-vector space which is also a Lie ring containing $R$ and $K$ as Lie ideals with suitable relations. (The enveloping ring $U(V, R, K)$ is a finite PBW extension of $R$ when $\operatorname{dim}_{K}(V / R)$ is finite.) Conversely, a PBW extension $R\left[\theta_{1}, \ldots, \theta_{n}\right]$ is a universal enveloping ring provided $R$ contains a central subfield $K$, invariant under each $\left[\theta_{i},-\right]$, such that each $\left[\theta_{l}, \theta_{j}\right]$ lies in the $K$-vector space $V=R+K \theta_{1}+\cdots+K \theta_{n}$. In fact, if we amend Passman's definition to allow $K$ to be any central subring of $R$ while also requiring that $V / R$ be a free left $K$-module, then one can show in general that

$$
R\left[\theta_{1}, \ldots, \theta_{n}\right]=U\left(R+K \theta_{1}+\cdots+K \theta_{n}, R, K\right)
$$

where $K$ is the center of $R$.

Returning to a general PBW extension $T=R\left[\theta_{1}, \ldots, \theta_{n}\right]$, we denote an ordered monomial $\theta_{1}^{i(1)} \theta_{2}^{\prime(2)} \cdots \theta_{n}^{i(n)}$ by $\theta^{I}$ where $I=(i(1), \ldots, i(n))$, and we define the total order of such a monomial in the usual way, namely as $|I|=i(1)+\cdots+i(n)$. Thus we can define the total order of any element of $T$. Using (b) and (c), it is not hard to check that for any $I$ and any $r \in R$ we have

$$
\theta^{I} r=r \theta^{I}+\text { (terms of lower total order). }
$$

The ring $T$ can be filtered by total order, and the associated graded ring of $T$ with respect to this filtration is the ordinary polynomial ring $R\left[\theta_{1}, \ldots, \theta_{n}\right]$. It now follows from standard arguments that if $R$ is either right or left noetherian, so is $T$. (See for example [14, Chapter V, §3]). More generally, if $M$ is any $R$-module, then $M$ is noetherian as an $R$-module if and only if $M \otimes_{R} T$ is noetherian as a $T$-module.

In order to define leading coefficients, we need a more delicate notion of order, which requires us to order $n$-tuples of nonnegative integers. We do so via the Dixmier ordering, namely we order $n$-tuples first by total order, then among $n$-tuples of the same total order we order them lexicographically, with the convention that $\theta_{1}>\cdots>\theta_{n}$. This ordering on $\left(\mathbb{Z}^{+}\right)^{n}$ gives an ordered set order-isomorphic to the natural numbers with their usual ordering; in particular, any $n$-tuple of nonnegative integers has only finitely many predecessors. (This ordering is discussed in $[3, \S 2.6]$.) We now define the monomial order of any nonzero element 
$t \in T$ as the largest $n$-tuple $I$ such that the coefficient of $\theta^{I}$ in $t$ is nonzero, and we define the leading coefficient of $t$ to be the coefficient $\lambda(t)$ of $\theta^{I}$ in $t$. We may define the monomial order of the zero element of $T$ as $(-\infty, \ldots,-\infty)$, and we may define its leading coefficient to be 0 . Monomial orders and leading coefficients are defined in the same manner for induced modules $M \otimes_{R} T$.

One can check that if $s, t$ are nonzero elements of $T$, then either $\lambda(s) \lambda(t)=\lambda(s t)$ or $\lambda(s) \lambda(t)=0$, and in the former case the monomial order of $s t$ is the sum of the monomial orders of $s$ and $t$, while in the latter case it is strictly less than this sum. It is now easy to see that we can duplicate the proof of Lemma 1.1 to obtain the following result.

LEMMA 5.1. Let $T=R\left[\theta_{1}, \ldots, \theta_{n}\right]$ be a $P B W$ extension of a ring $R$. If $N \leq{ }_{r} M$ are right $R$-modules, then $N \otimes_{R} T \leq{ }_{r} M \otimes_{R} T$ as $R$-modules and hence also as $T$-modules.

Since a minimal order argument is all that is involved in the proof of Lemma 3.1, it can be extended to the following result.

LEMMA 5.2. Let $T=R\left[\theta_{1}, \ldots, \theta_{n}\right]$ be a $P B W$ extension of a ring $R$, let $M$ be a right $R$-module, and let $A$ be a nonzero T-submodule of $M \otimes_{R} T$. If $x$ is a nonzero element of $A$ of minimal monomial order, then $\operatorname{ann}_{T}(x)=$ $\operatorname{ann}_{R}(\lambda(x)) T$. Thus $x T \cong \lambda(x) R \otimes_{R} T$ as right $T$-modules.

Recall that a nonzero right $R$-module $M$ is compressible if every nonzero submodule of $M$ contains an isomorphic copy of $M$. If $M$ is compressible and either $M$ is a noetherian module or $R$ is a right noetherian ring, then $M$ contains a uniform submodule, from which it follows that $M$ is uniform. Lemma 5.2 enables us to extend the result of $[10$, Lemma 2.1] for an ordinary differential operator ring to the case of a PBW extension.

Corollary 5.3. Let $T=R\left[\theta_{1}, \ldots, \theta_{n}\right]$ be a $P B W$ extension of a ring $R$ and let $M$ be a compressible right $R$-module. Then $M \otimes_{R} T$ is a compressible right $T$-module. Thus if either $R$ is a right noetherian ring or $M$ is a noetherian module, $M \otimes_{R} T$ is a uniform right T-module.

Proof. If $A$ is a nonzero submodule of $M \otimes_{R} T$ and $x \in A$ is as in the statement of Lemma 5.2, then $A$ contains an isomorphic copy of the module $\lambda(x) R \otimes_{R} T$. As $M$ is compressible, $\lambda(x) R$ contains a submodule isomorphic to $M$. Thus $A$ contains an isomorphic copy of $M \otimes_{R} T$. 
We can now generalize Proposition 1.5, provided we add a noetherian hypothesis.

Proposition 5.4. Let $T=R\left[\theta_{1}, \ldots, \theta_{n}\right]$ be a $P B W$ extension of a ring $R$ and let $M$ be a nonsingular right $R$-module. If either $R$ is a right noetherian ring or $M$ is a noetherian module, then

$$
\operatorname{rank}_{R}(M)=\operatorname{rank}_{T}\left(M \otimes_{R} T\right) .
$$

Proof. Either noetherian hypothesis implies that every nonzero submodule of $M$ contains a uniform noetherian submodule. This in turn implies that $M$ contains an essential submodule $N$ which is a direct sum of uniform noetherian submodules. Since $M$ is nonsingular, $N \leq_{r} M$, and so by Lemma 5.1, $N \otimes_{R} T \leq_{r} M \otimes_{R} T$. Hence, $\operatorname{rank}_{T}\left(N \otimes_{R} T\right)=$ $\operatorname{rank}_{T}\left(M \otimes_{R} T\right)$.

Thus we need only show that if $M$ is a nonsingular uniform noetherian module, then $M \otimes_{R} T$ is uniform. We know that $M \otimes_{R} T$ is noetherian, and so it contains a uniform submodule $A$. Let $x$ be a nonzero element of $A$ of minimal monomial order. Then by Lemmas 5.1 and 5.2 we see that

$$
x T \cong \lambda(x) R \otimes_{R} T \leq{ }_{r} M \otimes_{R} T .
$$

Since $x T$ is uniform, this implies that $M \otimes_{R} T$ is uniform.

We next use Lemma 5.2 to show that nonsingularity is preserved for induced modules. Note that the example in the introduction to $\$ 4$ gives a ring $R$ with nonzero singular ideal and a differential operator ring $T=R[\theta ; \delta]$ which is a prime noetherian ring, and hence is nonsingular, which shows that the complete converse to this is false.

Proposition 5.5. Let $T=R\left[\theta_{1}, \ldots, \theta_{n}\right]$ be a $P B W$ extension of a ring $R$ and let $M$ be a right $R$-module. If $M_{R}$ is nonsingular, then $\left(M \otimes_{R} T\right)_{T}$ is nonsingular. Conversely, if $R_{R}$ is nonsingular and $\left(M \otimes_{R} T\right)_{T}$ is nonsingular, then $M_{R}$ is nonsingular.

Proof. Assume that $M_{R}$ is nonsingular, and let $A$ be the singular submodule of $M \otimes_{R} T$. If $A \neq 0$, let $x \in A$ be nonzero with minimal monomial order. Then

$$
\operatorname{ann}_{T}(x)=\operatorname{ann}_{R}(\lambda(x)) T,
$$

and since $M$ is nonsingular, there is a nonzero right ideal $I$ of $R$ with $\operatorname{ann}_{R}(\lambda(x)) \cap I=0$. Therefore

$$
\operatorname{ann}_{R}(\lambda(x)) T \cap I T=0,
$$


and so $\operatorname{ann}_{T}(x)$ is not an essential right ideal of $T$, contrary to the definition of $A$. Thus $A=0$.

Now suppose that $R_{R}$ and $\left(M \otimes_{R} T\right)_{T}$ are nonsingular, and let $m$ be an element of $M$ with $I=r$-ann ${ }_{R}(m)$. If $I$ is an essential right ideal of $R$, then $I_{R} \leq{ }_{r} R_{R}$, and so $I T_{T} \leq{ }_{r} T_{T}$. Since $(m \otimes 1) I T=0$, this implies that $m=0$. Thus $M_{R}$ is nonsingular.

We are now ready to carry over the results on rank from $\$ 2$. Note that since each $\left[\theta_{i}, R\right] \subseteq R$, there is a derivation $\delta_{i}$ on $R$ such that $\left[\theta_{i}, r\right]=\delta_{i}(r)$ for all $r \in R$. Let $\Delta=\left\{\delta_{1}, \ldots, \delta_{n}\right\}$. If $I$ is an ideal of $R$ which is $\Delta$-invariant (that is, invariant under each derivation in $\Delta$ ), it is not hard to see that $I T=T I$ is an ideal of $T$ with $I T \cap R=I$. Thus $R / I$ embeds in $T / I T$, and we observe that $T / I T$ is a PBW extension of $R / I$.

Proposition 5.6. Let $T=R\left[\theta_{1}, \ldots, \theta_{n}\right]$ be a $P B W$ extension of a right noetherian ring $R$, let $\Delta$ be the set of derivations on $R$ induced by $\theta_{1}, \ldots, \theta_{n}$, and let $M$ be a tame right $R$-module such that each member of $\operatorname{ass}(M)$ is $\Delta$-invariant. Then $\operatorname{rank}_{R}(M)=\operatorname{rank}_{T}\left(M \otimes_{R} T\right)$.

Proof. As in the proof of Proposition 2.1, we may reduce to the case where $M=E$ is the injective hull of a uniform right ideal $U$ of some factor ring $R / P$, with $P$ a $\Delta$-invariant prime ideal of $R$. We set $E_{0}=$ $\operatorname{ann}_{E}(P)$, so that $E_{0}$ is the $(R / P)$-injective hull of $U$. Thus $E_{0}$ is torsionfree and uniform as an $(R / P)$-module, and so by Proposition 5.4, the module

$$
E_{0} \otimes_{R / P}(T / P T) \cong E_{0} \otimes_{R} T
$$

is uniform as a right $T$-module.

Thus we need only show that $E_{0} \otimes_{R} T \leq_{e} E \otimes_{R} T$. The proof is the same as the last paragraph of the proof of Proposition 2.1. If $E_{0} \otimes_{R} T$ is not essential in $E \otimes_{R} T$, we pick a nonzero $a \in E \otimes_{R} T$ of minimal monomial order such that $a T \cap\left(E_{0} \otimes_{R} T\right)=0$. Since $E_{0} \leq{ }_{e} E$, we may assume that the leading coefficient $\lambda(a)$ of $a$ is in $E_{0}$, so that $\lambda(a) P=0$. As $P$ is $\Delta$-invariant, it follows that for $I$ the monomial order of $a$, we have $\left(\lambda(a) \otimes \theta^{I}\right) P=0$. Now one gets $a P=0$ by minimality and from this it follows that each coefficient of $a$ is annihilated by $P$. Thus $a \in E_{0} \otimes T$, contradicting our choice of $a$.

TheOREM 5.7. Let $T=R\left[\theta_{1}, \ldots, \theta_{n}\right]$ be a $P B W$ extension of a right noetherian ring $R$ which has no $\mathbb{Z}$-torsion. If $R$ is tame as a right module over itself, then $\operatorname{rank}_{R}(R)=\operatorname{rank}_{T}(T)$. 
Proof. This follows immediately from Lemma 2.2 and Proposition 5.6 .

As in Corollary 2.4, there are some cases in which $R_{R}$ is automatically tame and so Theorem 5.7 immediately applies. As noted before, the annihilator condition is satisfied if $R$ is right and left noetherian.

Corollary 5.8. Let $T=R\left[\theta_{1}, \ldots, \theta_{n}\right]$ be a $P B W$ extension of a right noetherian ring $R$ which has no $\mathbb{Z}$-torsion. If $R$ is right fully bounded or $R$ has the d.c.c. on right annihilators, then $\operatorname{rank}_{R}(R)=\operatorname{rank}_{T}(T)$.

6. Reduced rank. When studying modules over a prime noetherian ring, often one is interested only in the torsionfree rank of a module, as opposed to its uniform rank. Over general noetherian rings, this leads to the notion of reduced rank, which turns out to behave better than uniform rank when inducing up to differential operator rings. We prove in this section that the reduced rank of an arbitrary module $M$ over a right noetherian ring $R$ remains the same when $M$ is induced up to a PBW extension of $R$, even in positive characteristic.

Recall that if $R$ is a semiprime right noetherian ring with right Goldie quotient ring $Q$ and $M$ is a right $R$-module, the reduced rank (or torsionfree rank) of $M$ is defined to be the uniform rank (equivalently, the composition series length) of the right $Q$-module $M \otimes_{R} Q$. If $R$ is an arbitrary right noetherian ring with prime radical $N$ and $M$ is a right $R$-module, the reduced rank, $\rho_{R}(M)$, of $M$ is defined as follows. Let

$$
M=M_{0} \geq M_{1} \geq \cdots \geq M_{k}=0
$$

be a chain of submodules of $M$ such that $M_{t-1} N \subseteq M_{i}$ for each $i$. (For example, we may take $M_{i}=M N^{i}$ when $N^{k}=0$.) Then $\rho_{R}(M)$ is the sum of the reduced ranks of the $(R / N)$-modules $M_{i-1} / M_{l}$. One checks that this definition is independent of the chain chosen, and that the reduced rank is additive on short exact sequences. (See [1, Chapter 2].)

The following lemma gives some additional properties of reduced rank that we will need.

LEMMA 6.1. Let $R$ be a right noetherian ring with prime radical $N$ and let $M$ be a right $R$-module.

(a) If $C$ is a right Ore set in $R$ which is contained in $\mathscr{C}(N)$, then

$$
\rho_{R}(M)=\rho_{R C^{-1}}\left(M \otimes_{R} R C^{-1}\right) .
$$

(b) If $I$ is an ideal of $R$ such that all the prime ideals of $R$ minimal over $I$ are minimal primes of $R$ and such that $M I=0$, then $\rho_{R}(M)=\rho_{R / I}(M)$. 
Proof. (a) Since $C$ is right Ore and is contained in $\mathscr{C}(N)$, it is well-known that $N R C^{-1}$ is the prime radical of $R C^{-1}$. Since localization is exact, it is enough to show that if $M N=0$, then

$$
\rho_{R}(M)=\rho_{R C^{-1}}\left(M \otimes_{R} R C^{-1}\right) .
$$

We may clearly assume without loss of generality that $N=0$ and hence that $C$ consists of regular elements.

Let $Q$ be the right Goldie quotient ring of $R$. Then $Q$ is also the right Goldie quotient ring of $R C^{-1}$, and

$$
M \otimes_{R} Q \cong\left(M \otimes_{R} R C^{-1}\right) \otimes_{R C^{-1}} Q
$$

(as right $Q$-modules). The equality of the reduced ranks follows from this isomorphism.

(b) Let $K$ be the prime radical of $I$, that is, the intersection of all prime ideals of $R$ containing $I$. Then $K / I$ is nilpotent in $R / I$, so there is a power of $K$ contained in $I$. Since $M I=0$, it follows that there is a chain of submodules of $M$ such that each factor is annihilated by $K$. Thus we may reduce to the case where $M K=0$.

Let $Q$ be the right Goldie quotient ring of $R / N$. Note that $Q / K Q$ is the right Goldie quotient ring of $R / K$ (because $K$ is the intersection of some of the minimal prime ideals of $R$ ). Since $M K=0$, we have

$$
M \otimes_{R / N} Q \cong M \otimes_{R / K}(Q / K Q)
$$

(as right $Q$-modules). The required equality follows from this isomorphism.

Since a right noetherian ring $R$ has only finitely many minimal prime ideals and their product is contained in the prime radical $N$, part (b) of Lemma 6.1 implies that we may compute $\rho_{R}(M)$ by taking a finite chain

$$
M=M_{0} \geq M_{1} \geq \cdots \geq M_{k}=0
$$

of submodules for which each $M_{i-1} / M_{i}$ is annihilated by a minimal prime $P(i)$ and getting

$$
\rho_{R}(M)=\rho_{R / P(1)}\left(M_{0} / M_{1}\right)+\cdots+\rho_{R / P(k)}\left(M_{k-1} / M_{k}\right) .
$$

We also need some information about prime ideals in a PBW extension $T=R\left[\theta_{1}, \ldots, \theta_{n}\right]$. As in the previous section, we use $\delta_{i}$ to denote the restriction of the derivation $\left[\theta_{i},-\right]$ to $R$, and we set $\Delta=\left\{\delta_{1}, \ldots, \delta_{n}\right\}$. We define a $\Delta$-invariant ideal $I$ of $R$ to be $\Delta$-prime if whenever a product of two $\Delta$-invariant ideals is contained in $I$, one of the ideals is contained in 
I. The first result below has been proved by Chin [2, Theorem 2.7] for a class of rings close to our PBW extensions, and so we merely sketch the main points of the proof - the reader is referred to Chin's paper for more details.

Proposition 6.2. Let $T=R\left[\theta_{1}, \ldots, \theta_{n}\right]$ be a $P B W$ extension of a ring $R$ and let $I$ be a $\Delta$-invariant ideal of $R$. Then $I$ is a $\Delta$-prime ideal of $R$ if and only if IT is a prime ideal of $T$.

Proof. Since $I T=T I$ is an ideal of $T$, we may pass to the PBW extension $R / I \subseteq T / I T$, and hence we may assume that $I=0$. If $R$ is not $\Delta$-prime, then clearly $T$ is not prime.

Thus we may assume $R$ is $\Delta$-prime and we need to show that if $A, B$ are nonzero ideals of $T$ then $A B \neq 0$. Let $n$ be any nonnegative integer. We define $B_{n}$ to be the ideal of $R$ generated by all elements of $R$ which are coefficients of some monomial of total order $n$ which appears as one of the terms of an element of $B$ of total order $n$. Bracketing such a monomial $r \theta^{I}$ with a $\theta_{j}$ will produce terms of the form $\delta_{j}(r) \theta^{I}$ and/or $r s \theta^{J}$ where $|J| \leq|I|$. Since the only derived coefficients occur with the same monomial order $I$ as the term they came from, the ideal $B_{n}$ is $\Delta$-invariant.

Now let $a$ be a nonzero element of $A$ of minimal monomial order and let $n$ be a nonnegative integer such that $B_{n} \neq 0$. If $a B=0$, Lemma 5.2 implies that

$$
B \subseteq r-\operatorname{ann}_{T}(a)=r-\operatorname{ann}_{R}(\lambda(a)) T .
$$

Thus $\lambda(a) B_{n}=0$ and so $l$-ann ${ }_{R}\left(B_{n}\right) \neq 0$. Since $B_{n}$ is nonzero and $\Delta$-invariant, this last fact contradicts $R$ being $\Delta$-prime. Therefore $a B \neq 0$ and so $A B \neq 0$.

We need one more set of facts about PBW extensions of right noetherian rings.

Proposition 6.3. Let $T=R\left[\theta_{1}, \ldots, \theta_{n}\right]$ be a PBW extension of a right noetherian ring $R$.

(a) If $T$ is prime, then $R$ has a (unique) nilpotent prime ideal $P$. Furthermore, if $C=\mathscr{C}_{R}(0)$, then also $C=\mathscr{C}_{R}(P)$ and $C$ is a right Ore set of regular elements in both $R$ and $T$. The ring $R C^{-1}$ is a right artinian ring with unique prime ideal $P R C^{-1}$ and $T C^{-1}$ is a $P B W$ extension of $R C^{-1}$, with the same basis. Also, if we let $\Delta^{\prime}$ be the set of derivations on $R C^{-1}$ induced by $\delta_{1}, \ldots, \delta_{n}$, then $R C^{-1}$ has no nontrivial $\Delta^{\prime}$-invariant ideals.

(b) If $T$ is prime and $R$ has no $\mathbb{Z}$-torsion, then $R$ is prime. 
Proof. The results about $R$ follow from [6] or [15, Theorem 2.2 and Lemma 2.1]. (The results in [15] are stated for a single derivation only, but the proofs are easily generalized.) Using leading coefficients it is easy to see that $C$ consists of regular elements in $T$. The fact that $C$ satisfies the right Ore condition in $T$ follows from the fact that $T$ is generated as a ring by the elements of $R$ and $\theta_{1}, \ldots, \theta_{n}$ and from the observation that for any $c \in C$ we have

$$
c\left(d \theta_{i}+r+\delta_{i}(d)\right)=\theta_{i} c d,
$$

where $c r=\delta_{i}(c) d$ with $r \in R$ and $d \in C$. Using the standard properties of Ore localizations, one can now check that $T C^{-1}$ is indeed a PBW extension of $R C^{-1}$.

We can now prove the reduced rank equality.

THEOREM 6.4. Let $T=R\left[\theta_{1}, \ldots, \theta_{n}\right]$ be a $P B W$ extension of a right noetherian ring $R$, and let $M$ be a right $R$-module. Then $\rho_{R}(M)=$ $\rho_{T}(M \otimes d R T)$.

Proof. Choose submodules $M=M_{0} \geq M_{1} \geq \cdots \geq M_{k}=0$ such that each $M_{i-1} / M_{i}$ is annihilated by a minimal prime ideal; then $\rho_{R}(M)$ is the sum of the values $\rho_{R}\left(M_{i-1} / M_{i}\right)$. Since $T$ is flat as a left $R$-module, $\rho_{T}\left(M \otimes_{R} T\right)$ is the sum of the values $\rho_{T}\left(\left(M_{i-1} / M_{i}\right) \otimes_{R} T\right)$. Thus, we may suppose that $M P=0$ for some minimal prime ideal $P$ of $R$.

Let $I$ be the largest $\Delta$-invariant ideal contained in $P$. It is easy to see that $I$ is a $\Delta$-prime ideal, and so Proposition 6.2 implies that $I T$ is prime in $T$. Furthermore, Proposition 6.3 implies that some power of $P$ is contained in $I$, and so $P$ is the unique prime minimal over $I$. If $Q$ is any prime ideal of $T$ contained in $I T$, then $J=Q \cap R$ is a $\Delta$-prime ideal contained in $I$. By Proposition 6.3 there is a prime ideal $Q_{0}$ of $R$ with $Q_{0}^{n} \subseteq J \subseteq P$ for some $n$. Since $P$ is minimal, this implies $P=Q_{0}$, and so $I^{n} \subseteq P^{n} \subseteq J$. Thus $I=J$ (because $J$ is $\Delta$-prime), whence $I T=Q$. This shows that $I T$ is a minimal prime ideal of $T$.

Since $M I=0$ and $I$ is $\Delta$-invariant, $\left(M \otimes_{R} T\right) I T=0$. By Lemma 6.1(b), $\rho_{R}(M)=\rho_{R / I}(M)$ and

$$
\rho_{T}\left(M \otimes_{R} T\right)=\rho_{T / I T}\left(M \otimes_{R} T\right)=\rho_{T / I T}\left(M \otimes_{R / I} T / I T\right) .
$$

Thus we may pass to the PBW extension $R / I \subseteq T / I T$, and hence assume that $I=0$. Now $T$ is a prime ring, and $P$ is the prime radical of $R$. 
Using Proposition 6.3(a) and Lemma 6.1(a), we may localize at the set $C$ of regular elements of $R$ and thus assume that $R$ is a right artinian ring. Since $M P=0$ and $R / P$ is a simple artinian ring, $M$ is a direct sum of simple right $(R / P)$-modules, and hence we may reduce to the case where $M$ is simple. Then $M$ is uniform and torsionfree as an $(R / P)$-module, and so $\rho_{R}(M)=1$. By Corollary 5.3, $M \otimes_{R} T$ is a uniform $T$-module. Since $M$ is (up to isomorphism) the unique simple right $R$-module and $R$ is right artinian, $M$ embeds in $R_{R}$. This implies that $M \otimes_{R} T$ embeds in the prime right noetherian ring $T$, and so is torsionfree as a $T$-module. Thus $\rho_{T}\left(M \otimes_{R} T\right)=1$, which completes the proof.

\section{REFERENCES}

[1] A. W. Chatters and C. R. Hajarnavis, Rings with Chain Conditions, Research Notes in Mathematics No. 44, Pitman, Boston, 1980.

[2] W. Chin, Prime ideals in differential operator rings and crossed products of infinite groups, J. Algebra, 106 (1987), 78-104.

[3] J. Dixmier, Enveloping Algebras, North-Holland Mathematical Library Vol. 14, North-Holland, Amsterdam, 1977.

[4] G. L. Fel'dman, The global homological dimension of general rings of differential operators, (Russian), Uspekhi Mat. Nauk, 29 no. 5 (1974), 245-246. , Global dimension of rings of differential operators (Russian), Trudy Moskov. Mat. Obshch., 41 (1980), 121-143 [Translation: Trans. Moscow Math. Soc., (1982) Issue 1, 123-147].

[6] J. R. Fisher, A Goldie theorem for differentiably prime rings, Pacific J. Math., 58 (1975), 71-77.

[7] K. R. Goodearl, Nonsingular Rings and Modules, Pure and Applied Mathematics Vol. 33, Marcel Dekker, New York, 1976.

[8] K. R. Goodearl, T. J. Hodges, and T. H. Lenagan, Krull and global dimensions of Weyl algebras over division rings, J. Algebra, 91 (1984), 334-359.

[9] K. R. Goodearl and T. H. Lenagan, Krull dimension of differential operator rings. III: Noncommutative coefficients, Trans. Amer. Math. Soc., 275 (1983), 833-859.

[10] K. R. Goodearl and R. B. Warfield, Jr., Krull dimension of differential operator rings, Proc. London Math. Soc. (3), 45 (1982), 49-70.

[11] _ Primitivity in differential operator rings, Math. Z., 180 (1982), 503-523.

[12] P. Grzeszczuk, Goldie dimension of differential operator rings, Comm. Algebra, (to appear).

[13] G. Hochschild, B. Kostant, and A. Rosenberg, Differential forms on regular affine algebras, Trans. Amer. Math. Soc., 102 (1962), 383-408.

[14] N. Jacobson, Lie Algebras, Wiley-Interscience, New York, 1962.

[15] D. A. Jordan, Noetherian Ore extensions and Jacobson rings, J. London Math. Soc. (2), 10 (1975), 281-291.

[16] J. C. McConnell, Representations of solvable Lie algebras and the Gelfand-Kirillov conjecture, Proc. London Math. Soc. (3), 29 (1974), 453-484.

[17] The global dimension of rings of differential operators, in Sem. $d^{\prime}$ 'Alg. P. Dubreil, pp. 189-197, Lecture Notes in Math. No. 641, Springer-Verlag, Berlin, 1978. 
[18] D. S. Passman, Prime ideals in enveloping rings, Trans. Amer. Math. Soc., (to appear).

[19] D. Quinn, Embeddings of differential operator rings, and Goldie dimension, Proc. Amer. Math. Soc., (to appear).

[20] G. S. Rinehart, Differential forms on general commutative algebras, Trans. Amer. Math. Soc., 108 (1963), 195-222.

[21] R. C. Shock, Polynomial rings over finite-dimensional rings, Pacific J. Math., 42 (1972), 251-257.

[22] G. Sigurdsson, Differential operator rings whose prime factors have bounded Goldie dimension, Arch. Math. (Basel), 42 (1984), 348-353.

Received July 27, 1986.

UNIVERSITY OF UTAH

Salt Lake City, UT 84112 



\section{PACIFIC JOURNAL OF MATHEMATICS EDITORS}

\author{
V. S. VARADARAJAN \\ (Managing Editor) \\ University of California \\ Los Angeles, CA 90024 \\ Herbert Clemens \\ University of Utah \\ Salt Lake City, UT 84112 \\ R. FINN \\ Stanford University \\ Stanford, CA 94305
}

ROBION KIRBY

University of California

Berkeley, CA 94720

C. C. MOORE

University of California

Berkeley, CA 94720

HAROLD STARK

University of California, San Diego

La Jolla, CA 92093

\section{ASSOCIATE EDITORS}
R. ARENS
E. F. BECKENBACH
B. H. NEUMANN
F. WOLF
K. YOSHIDA (1906-1982)

\section{SUPPORTING INSTITUTIONS}
UNIVERSITY OF ARIZONA
UNIVERSITY OF OREGON
UNIVERSITY OF BRITISH COLUMBIA UNIVERSITY OF SOUTHERN CALIFORNIA
CALIFORNIA INSTITUTE OF TECHNOLOGY
UNIVERSITY OF CALIFORNIA
STANFORD UNIVERSITY
MONTANA STATE UNIVERSITY
UNIVERSITY OF HAWAII
UNIVERSITY OF NEVADA, RENO
UNIVERSITY OF TOKYO
NEW MEXICO STATE UNIVERSITY
UNIVERSITY OF UTAH
OREGON STATE UNIVERSITY
WASHINGTON STATE UNIVERSITY UNIVERSITY OF WASHINGTON 


\section{Pacific Journal of Mathematics}

\section{Vol. 131, No. $1 \quad$ November, 1988}

Tomek Bartoszynski, On covering of real line by null sets $\ldots \ldots \ldots \ldots \ldots 1$

Allen Davis Bell and Kenneth R. Goodearl, Uniform rank over differential operator rings and Poincaré-Birkhoff-Witt extensions $\ldots \ldots \ldots \ldots \ldots 13$

Brian Boe, Thomas Jones Enright and Brad Shelton, Determination of the intertwining operators for holomorphically induced representations of Hermitian symmetric pairs

Robert F. Brown, Topological identification of multiple solutions to

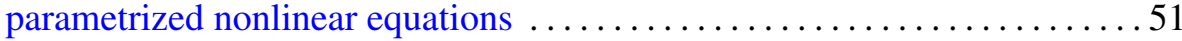

Marc R. M. Coppens, Weierstrass points with two prescribed nongaps ......71

Peter Larkin Duren and M. Schiffer, Grunsky inequalities for univalent functions with prescribed Hayman index .................... 105

Robert Greene and Hung-Hsi Wu, Lipschitz convergence of Riemannian manifolds

Kathryn E. Hare, Arithmetic properties of thin sets

Neal I. Koblitz, Primality of the number of points on an elliptic curve over a

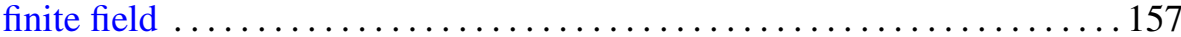

Isabel Dotti de Miatello and Roberto Jorge Miatello, Transitive isometry groups with noncompact isotropy

Raymond A Ryan, Weakly compact holomorphic mappings on Banach

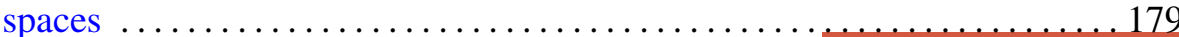

Tudor Zamfirescu, Curvature properties of typical convex surfaces 